\title{
HOMESHARE EUROPE. ALOJAMIENTO COMPARTIDO A CAMBIO DE AYUDA EN EUROPA
}

\author{
Ma ASUNCIÓN MARTÍNEZ ROMÁN* \\ ANNE-LOTTE KREICKEMEIER** \\ JOAQUINA MURCIA*** \\ HORTENSIA REDERO* \\ * Departamento de Trabajo Social y Servicios Sociales y Asociación Universitaria para \\ la Promoción del Voluntariado. Universidad de Alicante. \\ *** Fachhochschule. Darmstadt (Alemania). \\ ** Departamento de Trabajo Social y Servicios Sociales. Universidad de Alicante.
}

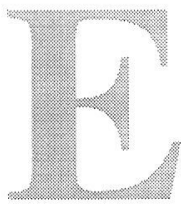

n este artículo se describen y analizan experiencias que contribuyen a promover la solidaridad intergeneracional entre personas mayores y estudiantes universitarios proporcionándoles opciones de ayudarse mutuamente para favorecer su desarrollo humano y social. Como desarrollo humano y social entendemos la propuesta de Naciones Unidas, por lo que partimos de las orientaciones contenidas en el Plan de Acción de la Cumbre Mundial de Desarrollo Social (Copenhague, 1995) y de la evaluación de los progresos realizada en la Asamblea General Extraordinaria de Ginebra en la que se reafirman los compromisos iniciales, invitando a todas las personas y a todos los países a sumarse para conseguir un mundo más justo y equitativo:

"Promover la integración social fomentando sociedades estables, seguras y justas, que estén basadas en la promoción y protección de todos los derechos humanos, así como en la nodiscriminación, la tolerancia, el respeto de la diversidad, la igualdad de oportunidades, la solidaridad, la seguridad y la participación de todas las personas, incluidos los grupos y las personas desfavorecidas y vulnerables" ( $4^{\circ}$ compromiso $\left.{ }^{1}\right)$.

Entre las actuaciones para conseguirlo se destaca la importancia de la participación en la vida social de todos y todas los/as ciudadanos/as, con especial referencia a la cooperación entre gobierno y sociedad civil, la contribución del voluntariado a la creación de sociedades que se

1 Naciones Unidas (2000) Nuevas iniciativas en pro del desarrollo social. A/RES/S-24/2. 
preocupen de las necesidades de sus diversos grupos, el apoyo a la familia en sus funciones de apoyo y "promover la plena integración y la continuación de las personas de edad en la sociedad como actores de pleno derecho en el proceso de desarrollo".

Una sociedad integrada e integradora, tal como la define Naciones Unidas, precisa "inversiones en instituciones sociales y para la promoción social, que se mejoren las redes de contacto y las relaciones sociales, se consiga el consenso y se aumente la capacidad de las personas, las organizaciones y las instituciones dentro de un marco propicio de cooperación mundial"2. Sin un entorno propicio no sólo será difícil la integración social sino que, además, tendremos personas y grupos en situación de vulnerabilidad que les colocará en riesgo de iniciar procesos de exclusión social. Situación de vulnerabilidad que no suele ser suficientemente considerada ni por las políticas públicas, cuando no se prima la prevención, ni por la sociedad que suele desconocer la vulnerabilidad en que viven personas y grupos por lo que no demanda atención. Lo cierto es que las personas mayores y los jóvenes son grupos sociales vulnerables y en riesgo de exclusión social por razón de edad y un modo de contribuir a reducir este riesgo es promover un mejor conocimiento de los obstáculos que encuentran para su desarrollo humano, fomentar la solidaridad y la participación social.

La eliminación de los obstáculos que las personas mayores y los jóvenes encuentran para su desarrollo humano es tarea de todos, del gobierno, de la sociedad y, por supuesto, de las propias personas interesadas, todos somos corresponsables. Desde esta perspectiva, las experiencias de ayuda mutua que vamos a comentar conciben la ayuda mutua (que se concreta en un intercambio de apoyo afectivo, social y económico) como un medio de ejercer esa responsabilidad compartida desde una posición de igualdad, de relaciones simétricas que permiten encontrar respuestas a necesidades personales de modo solidario mediante un intercambio, a través de actuaciones de mediación realizadas por terceros que intervienen como intermediarios, con el objetivo de promover la solidaridad intergeneracional. En ningún modo se trata de sustituir la responsabilidad de las políticas públicas ni la responsabilidad de la sociedad ni la de las familias o redes informales, no sustituye a otros recursos posibles sino que los complementa. Son experiencias innovadoras que contribuyen a prevenir la exclusión social de las personas mayores y jóvenes.

2 Naciones Unidas. Consejo Económico y Social (1998). Promoción de la integración y participación social de toda la gente, incluidos los grupos y personas desfavorecidos y vulnerables. Comisión de Desarrollo Social. E/CN.5/1998/2 


\section{ALOJAMIENTO COMPARTIDO A CAMBIO DE AYUDA EN EUROPA}

El Proyecto "Homeshare Europe", cofinanciado por la Comisión Europea (diciembre 1999 - noviembre 2000), ha permitido conocer experiencias que se están desarrollando en Europa en el ámbito del Alojamiento Compartido. El Proyecto ha sido coordinado por The Foundation for European Initiatives (Reino Unido) y han participado Homeshare International (Reino Unido), la Asociación Universitaria para la Promoción del Voluntariado (Universidad de Alicante), Fachhoschule Darmstadt y Cruz Roja de Darmstadt (Alemania) y Odyssee (Holanda).

Alojamiento Compartido es un concepto muy amplio. Al hablar de Alojamiento Compartido a Cambio de Ayuda, nos referimos a una situación de intercambio entre iguales, en el que una persona mayor ofrece alojamiento a un/una joven a cambio de un tipo de ayuda acordada previamente. Se trata de una modalidad de ayuda mutua en la que las dos partes implicadas manifiestan desear satisfacer unas necesidades ofreciendo algo a cambio. Estos programas se inician en Europa al comenzar la década de los 90, siendo los países pioneros Alemania y España, seguidos de Gran Bretaña y Austria. España destaca por ser el país con mayor número de programas.

En todos los países las personas mayores desean continuar viviendo en su propio domicilio en el que suelen tener espacio suficiente para más de una persona. Algunas de estas personas mayores necesitan, o podrían necesitar en el futuro próximo, algún tipo de ayuda para las tareas cotidianas y, en muchos casos, desean vivir en compañía de otra persona para disminuir su soledad. Por otra parte, muchos jóvenes manifiestan dificultades para satisfacer su necesidad de encontrar un alojamiento a un precio asequible. En este contexto, las experiencias de Alojamiento Compartido a Cambio de Ayuda realizan actuaciones de mediación entre las personas mayores y los jóvenes para ayudarles a encontrar la mejor respuesta a sus necesidades mediante un intercambio de ayuda mutua, tras una elección en total libertad. Con el tiempo, se ha ampliado la posibilidad de beneficiarse de este nuevo recurso a las personas que tienen discapacidades y la familias con un solo progenitor y con cargas familiares.

Las experiencias estudiadas muestran una gran diversidad, con una mayor similitud entre Alemania, Austria y España, lo que parece deberse al hecho de ser iniciativas surgidas de las Universidades con la intención de promover la ayuda mutua y la solidaridad intergeneracional. Las experiencias inglesas difieren en su origen y diseño. 
Por ser experiencias innovadoras hay que invertir tiempo en su promoción, siendo la mejor propaganda la que realizan las personas mayores y los jóvenes que han participado. Las actividades de mediación (selección, emparejamiento, inicio de la convivencia y seguimiento) deben ser realizadas por profesionales cualificados y, además, puede ser muy enriquecedor contar con la colaboración de personas mayores.

\section{LOS PROGRAMAS DE ALOJAMIENTO COMPARTIDO A CAMBIO DE AYUDA EN ESPAÑA}

Los programas de Alojamiento Compartido entre personas mayores y estudiantes universitarios se inician en Europa al comenzar la década de los 90, siendo los países pioneros Alemania y España, seguidos de Gran Bretaña y Austria. En España, el primer programa de alojamiento compartido entre personas mayores y estudiantes universitarios se inicia en 1991 en la Universidad de Granada, por iniciativa de dos trabajadoras sociales del gabinete de atención social al estudiante. Actualmente, España tiene 17 programas consolidados que se desarrollan en 28 provincias y están en su inicio en otras 4 provincias más ${ }^{3}$, resultando ser el país de Europa con mayor número de este tipo de programas que promueven la solidaridad intergeneracional a través del alojamiento compartido entre personas mayores y estudiantes universitarios. Hay que recordar que estos datos se refieren a un estudio realizado en el año 2000 , considerando los programas que llevaban más de dos años en vigor, por lo que actualmente su número será superior ${ }^{4}$.

\subsection{Quién gestiona los programas}

En España, los programas de alojamiento a cambio de ayuda son gestionados de diversos modos, siendo lo más frecuente un sistema mixto en el que una Universidad, una entidad pública o una entidad privada sin ánimo de lucro asume la responsabilidad principal con la coparticipación de una o varias entidades más. Una característica en

3 Cronológicamente: 1991: Granada. 1992-93: Sevilla, Cádiz-Jerez de la Frontera, Córdoba, Málaga, Alicante, León y Albacete. 1995-96: Almería, Jaén (no contesta cucstionario), Navarra (desaparecido), Donosita-San Sebastián, Murcia y Las Palmas de Gran Canaria. 1996-97: Valladolid (Palencia, Segovia y Soria), Madrid y Cataluña. 1997-98: Santiago de Compostela. 1998-99: Bilbao-Deusto.

4 Martínez Román, $\mathrm{M}^{x}$ A. y Murcia Rodríguez, J. "Programas de alojamiento compartido a cambio de ayuda en España", en Kreickemeier, A.L y Martínez Román, M $^{a}$ A. (Dirs) Alojamiento compartido a cambio de ayuda en Europa, Alicante, Universidad de Alicante, 2001, págs. 21-34. 
España es que en todos participa una Universidad y docentes y profesionales vinculados al Trabajo Social. Las entidades públicas implicadas son bien del gobierno local (Ayuntamiento, Diputación) o bien del gobierno autonómico (Consejerías de Comunidades Autónomas). En cuanto a las entidades privadas sin ánimo de lucro, se trata de asociaciones y organizaciones de voluntariado. También hay una entidad de ahorro que asume la gestión directamente en colaboración con Universidades y Ayuntamientos.

Las Universidades desarrollan estos programas como un medio de promover la solidaridad intergeneracional ya que pueden ofrecer información a los estudiantes sobre posibles alojamientos mediante un servicio de "bolsa de alojamiento" convencional. El servicio que presta la Universidad con esta bolsa de alojamiento se diferencia claramente de los Programas de ayuda mutua a los que nos referimos, cuando las personas mayores se inscriben en esta bolsa como potenciales alojadoras lo hacen con la finalidad de obtener un ingreso económico extra, mediante el alquiler de una habitación a los estudiantes y la Universidad simplemente ofrece una información sin más intervención posterior.

En cambio, los programas que nos ocupan vinculan el hecho de compartir un alojamiento a la oportunidad de convivir solidariamente con una persona mayor y a la promoción de un acercamiento generacional, todo ello basado en la ayuda mutua. Es decir, si cada una de las partes tiene carencias que puede satisfacer la otra parte y ambas partes tienen actitudes solidarias, pero se desconocen entre sí, la introducción de una figura mediadora puede crear un nuevo recurso social "no convencional". Este recurso beneficia tanto a las personas mayores, porque pueden permanecer en su domicilio a la vez que se sienten útiles (incluso rejuvenecidas) compartiendo su vivienda con estudiantes, como a los jóvenes que pueden disponer de un alojamiento a la vez que se sienten solidarios. Sin embargo, no se trata de una varita mágica que solucione todos los problemas de las personas mayores que quieren permanecer en su domicilio ni de los jóvenes que buscan alojamiento, en ambos casos es una modalidad selectiva por lo que no se debe valorar en términos cuantitativos sino cualitativos. Es una opción más entre otras, a las que nunca debe excluir.

\subsection{Perfil de las personas mayores alojadoras y de las/los estu- diantes}

El perfil de la persona que ofrece un alojamiento a cambio de ayuda es el de una mujer, viuda, mayor de 70 años (entre 60 y 90 años), de nivel socioeconómico medio y medio-bajo, que quiere vivir en su propio domicilio, puede cuidarse de modo independiente, pero tiene alguna di- 
ficultad que va en aumento debido a la edad. Sobre todo quiere compañía y tiene miedo a vivir sola, especialmente, miedo a estar sola por las noches.

Y el perfil de la persona que ofrece ayuda a cambio de alojamiento es el de una estudiante mujer, mayor de 25 años, que cursa primeros cursos o post-grado, con escasos ingresos, que busca alojamiento y está dispuesta a hacer compañía compatible con sus estudios.

\subsection{En qué consiste el intercambio de ayuda que se realiza}

Las personas mayores ofrecen alojamiento y reciben compañía y pequeñas ayudas en las tareas cotidianas, dependiendo del acuerdo previo entre ambas partes. Se insiste a las personas mayores en que esta modalidad de convivencia se basa en la ayuda mutua y no es un medio para obtener un pequeño ingreso económico adicional. Sin embargo, para que todas las personas mayores puedan beneficiarse de este programa, cuando una persona mayor tiene bajos ingresos y no puede afrontar los gastos que le ocasionaría otra persona en el hogar, puede recibir una pequeña cuantía económica como compensación de esos pequeños gastos adicionales de agua, electricidad y gas. Esta ayuda económica puede quedar a cargo del propio estudiante o ser alguna entidad social pública o privada la que financie estos gastos. Un caso especial es la Diputación Foral de Donostia-San Sebastián que, tras cinco años de experiencia, acaba incluyendo el programa de convivencia de estudiantes con personas mayores en su oferta de recursos sociales, pudiendo percibir la persona mayor una ayuda económica para compensar al estudiante que le dedica más tiempo de compañía y cuidados especiales ${ }^{5}$.

Los/as estudiantes proporcionan compañía a las personas mayores a cambio del alojamiento (se suele garantizar acompañamiento las noches de la semana lectiva) y ayuda en pequeñas tareas cotidianas. Hay programas que ofrecen al estudiante otras pequeñas compensaciones como becas de comedor o ayudas económicas para los gastos de transporte, tasas académicas o compra de libros escolares. En algunos casos, los/as estudiantes abonan directamente a la persona mayor la parte proporcional de los gastos de agua, electricidad y gas. Hay algunos Programas que se ocupan también de personas mayores dependientes en los que los estudiantes han de comprometerse a proporcionar determinados cuidados a las personas mayores y reciben por ello una peque-

5 Decreto Foral 73/2000, de 18 de julio, por el que se regula el Programa de Convivencia de Estudiantes con Personas Mayores (B. O. De Guipúzcoa n 142, de 27 de julio de 2000). 
ña compensación económica que financia alguna entidad social pública o privada, si bien nunca se trata de una remuneración o salario.

En ningún caso las personas mayores ni estudiantes tienen que abonar cantidades económicas a las entidades responsables de los programas por el servicio recibido.

En todos los casos, los gastos de manutención son a cargo del estudiante alojado y se establece un periodo de prueba.

\subsection{Criterios de selección.}

Las personas mayores deben tener más de 60 años, desear compartir la vivienda y convivir con jóvenes, ofreciéndose mutua compañía y apoyo. No importa el nivel de ingresos económicos de las personas mayores si bien, en igualdad de condiciones, parece que se puede dar preferencia a las personas con menores ingresos económicos.

Los/as estudiantes deben estar matriculados en las Universidades relacionadas con los programas y desear compartir el alojamiento que le ofrece una persona mayor seleccionada por el programa a cambio de ofrecerle su compañía y/o cuidados. No importa el nivel de ingresos de los/as alumnos, si bien, en igualdad de condiciones, también se tiende a dar preferencia a aquellos/as con menores ingresos económicos familiares o sin derecho a alguna modalidad de beca para alojamiento.

Para todo ello se establece un acuerdo previo entre ambas partes. En todos los casos las entidades responsables de estos programas realizan tareas de mediación entre las posibles "parejas" que pueden llegar a convivir.

Se realizan entrevistas personales para seleccionar tanto a las personas mayores como a los/as estudiantes. En el caso de las personas mayores hay que realizar entrevistas en el domicilio para conocer las características de la vivienda. Una vez seleccionados los potenciales participantes se une a cada pareja y se inicia la convivencia con un periodo de prueba. Superado el periodo de prueba, se realiza un seguimiento durante todo el periodo temporal que dure la convivencia a fin de garantizar que se cumplen los objetivos del programa, actuando de mediadores en el caso de que surjan dificultades.

En la selección de las parejas intervienen mayoritariamente trabajadores sociales y en algunos programas también psicólogos (Albacete, Cataluña, Murcia, Santiago de Compostela y Sevilla). En cuanto al seguimiento de la convivencia, también se lleva a cabo mayoritariamente por trabajadores sociales. 


\subsection{Financiación actual del Programa}

Todas las Universidades contribuyen ofreciendo infraestructura básica, aportan también recursos humanos técnicos (Bilbao-Deusto, Granada, Las Palmas de Gran Canaria, Málaga, Murcia, Sevilla y Valladolid) y otros apoyos, como ayudas económicas puntuales o becas de comedor, transporte o libros (Alicante, Almería, Granada, Las Palmas de Gran Canaria, Murcia, Valladolid). Junto con las Universidades actúan como co-financiadoras otras entidades públicas o privadas: hay co-financiación de entidades públicas del gobierno autonómico (Andalucía, Valladolid), de la Administración Local (Albacete, Almería, Donostia-San Sebastián, Madrid, Murcia, Santiago de Compostela). En Las Palmas de Gran Canaria la Entidad co-financiadora es Cruz Roja.

Cuando la iniciativa y desarrollo del Programa se deben fundamentalmente a una entidad privada sin ánimo de lucro en colaboración con una Universidad, las entidades privadas aportan financiación propia y recursos humanos (técnicos con relación laboral y voluntarios), interviniendo también en la co-financiación del programa otras entidades públicas y entidades de ahorro, Caja de Ahorros del Mediterráneo, en el caso de Alicante, y, en Madrid, Caja Madrid. El Programa de Cataluña es financiado por Caixa-Cataluña en colaboración con Universidades y Ayuntamientos. Sólo un programa carece de ayuda financiera, aunque manifiesta estar gestionando financiación del gobierno autonómico (León).

\subsection{Obstáculos y apoyos encontrados}

Entre los obstáculos se señala que:

- Resulta difícil comenzar algo nuevo y sin precedentes. Hay que hacer mucho esfuerzo para difundirlo y captar potenciales personas alojadoras y alojables. Cuesta abrir camino. Durante su desarrollo, sigue siendo difícil la difusión de este recurso social, unas veces aumenta la demanda de estudiantes y no hay suficiente demanda de personas mayores y, otras veces, ocurre lo contrario. Se precisa mucha dedicación para el desarrollo de un programa de calidad y, generalmente, faltan más recursos humanos cualificados porque falta apoyo financiero.

- También hay muchas personas mayores que, a pesar de sentir soledad, no quieren compartir su vivienda o no tienen una vivienda adecuada aunque deseen compartirla. Hay casos en los que los hijos no aceptan que su madre comparta su domicilio con personas ajenas a la familia.

- Puede haber desconfianza mutua entre personas mayores y estu- 
diantes por desconocimiento intergeneracional y miedo de ambos a perder libertad. Hay diferencias de hábitos de vida cotidiana entre personas mayores y estudiantes.

- Suele ser difícil realizar emparejamientos entre diferentes sexos. Las señoras potenciales alojadoras suelen pedir una chica y suelen excluir a los varones, sin embargo, cuando los aceptan establecen muy buenas relaciones.

- Puede haber condicionamientos debidos a la estructura temporal del curso académico que resulten un inconveniente para la persona mayor.

Como facilitadores se señalan:

- Los recursos humanos que la entidad pone a disposición del programa, el apoyo de la Universidad y la coordinación entre entidades copartícipes en el programa.

- El apoyo de trabajadores sociales de los Servicios Sociales básicos y especializados.

- Una buena selección y un buen seguimiento garantiza el éxito de la convivencia.

- Hay que realizar una buena difusión del programa en los medios de comunicación por ser una experiencia innovadora y la mejor difusión es la que realizan los propios participantes en el programa, tanto personas mayores como estudiantes, que han tenido una buena experiencia.

\subsection{Algunos "consejos" para quien inicie nuevos programas}

- Invertir tiempo y esfuerzos en la difusión del programa y en la captación de potenciales personas interesadas. Es importante la colaboración de los medios de comunicación.

- Comprender que el programa es muy específico, siendo más importante lograr una convivencia duradera de las parejas que un gran número de ellas.

- Realizar una cuidadosa selección y emparejamiento entre las personas demandantes. No tener prisa y asegurarse de las posibilidades de éxito de la convivencia para evitar experiencias negativas en el inicio. Invertir todo el tiempo que sea necesario en la formacióninformación de las parejas para prevenir dificultades en la convivencia ayudándoles a aceptarse mutuamente.

- Realizar un seguimiento continuado de la convivencia ofreciendo una amplia franja horaria de atención por parte de los profesionales que ejecutan el programa. 
- Ampliar la oferta de compañía y apoyo, como personas alojadoras, a personas con discapacidades, enfermedades crónicas y padres/ madres con personas dependientes a cargo y jóvenes inmigrantes o queriendo acceder a un empleo.

- Que las personas potencialmente alojadoras y alojadas comprendan y acepten que es un programa de solidaridad y ayuda mutua, por lo que es indispensable la generosidad y la comprensión de ambas partes.

- Informarse sobre las experiencias que ya se han consolidado.

\section{COMPARACIÓN ENTRE LOS PROGRAMAS DE ALOJA- MIENTO COMPARTIDO A CAMBIO DE AYUDA DE ES- PAÑA, REINO UNIDO, ALEMANIA Y AUSTRIA?}

\subsection{Modos de financiación}

Las fuentes de financiación son muy heterogéneas tanto entre países como entre programas del mismo país. Generalmente, no hay una única entidad (pública o privada) que se responsabilice de la total financiación del programa, por el contrario, se establecen diferentes modalidades de acuerdos que implican contribuir a hacer viable el desarrollo del programa aportando alguno o varios de los siguientes tipos de recursos: económicos, humanos (profesionales técnicos y personas voluntarias) y también la infraestructura física y material necesaria (despachos, teléfonos, difusión, etc).

En el caso de Gran Bretaña, hay programas que fueron apoyados en su inicio financieramente por organizaciones privadas no lucrativas pero, actualmente, se autofinancian en parte con las aportaciones que reciben tanto de las personas alojadoras como de las alojadas, sin que ello cubra la totalidad del coste del programa. En España y Gran Bretaña hay programas que también se ocupan de personas mayores que necesitan cuidados especiales y, en estos casos, la persona alojada recibe una compensación por los cuidados extra, que puede estar financiada por los Servicios Sociales de la Administración pública o por una

- Más información: Ma Asunción Martínez Román. Asociación Universitaria para la Promoción del Voluntariado. Edificio de Ciencias Sociales. Universidad de Alicante. Apdo. Correos 99.03080 Alicante. E-mail: masun.martinez@ua.es.

7 Kreickemeier, A.L y Martínez Román, $M^{2}$ A. "Comparación entre los países", en Kreickemeier, A.L y Martínez Román, $\mathrm{M}^{a}$ A. (Dirs) Alojamiento compartido a cambio de ayuda en Europa. Alicante, Universidad de Alicante, 2001, págs. 49-57. 
entidad privada sin ánimo de lucro (por ejemplo, asociaciones de voluntariado y cajas de ahorro). Nos parece destacable que uno de los programas españoles se encuentre incluido entre los recursos sociales disponibles en la red pública de Servicios Sociales de su territorio como una de las modalidades posibles de acogimiento familiar.

Sin embargo, muchos de los programas no tienen suficiente financiación y manifiestan que con mayores recursos económicos podrían garantizar un mayor tiempo de dedicación por parte de profesionales cualificados y, de este modo, podrían incluir en el programa un mayor número de personas mayores y jóvenes y dedicarles una más amplia atención. Los profesionales de los Servicios Sociales públicos que están implicados en el desarrollo de estos programas remarcan que se trata de una muy buena alternativa para ayudar a las personas mayores a permanecer en sus domicilios y que la Administración pública debería potenciar su desarrollo apoyando con su financiación.

\subsection{Perfil y función de los coordinadores del programa}

En la mayoría de los programas los coordinadores son técnicos en Ciencias Sociales, principalmente trabajadores sociales. Su función es la siguiente:

- Asesorar a las personas interesadas en el "alojamiento compartido".

- Evaluar a las personas candidatas al programa.

- Poner en contacto y formar parejas entre personas propietarias interesadas en compartir su hogar, y personas interesadas en encontrar alojamiento.

- Realizar tareas de supervisión y seguimiento.

- Ofrecer el apoyo que garantice la estabilidad y afianzamiento del programa.

Además, serán quienes se encarguen de fomentar el desarrollo del programa. Para llevar a cabo esta tarea es necesario realizar un buen trabajo en relaciones públicas.

\subsection{Naturaleza del intercambio}

La idea básica de todos los programas es un intercambio basado en relaciones de reciprocidad en el que una parte ofrece alojamiento y la otra parte ofrece ayuda, pero las condiciones para que este intercambio tenga lugar varían considerablemente:

En Reino Unido -en términos generales-, la persona alojadora y la alojada pagan una cantidad mensual a la entidad que forma las parejas. 
La persona alojada ofrece además ayuda a la persona alojadora. Una ayuda que tiene distintas variaciones:

- En unos casos, se establecen 10 horas semanales de ayuda en tareas cotidianas, permaneciendo en la casa una media de 4 noches por semana y parte del fin de semana, además de pagar una cantidad a la organización.

- En otros, una ayuda a tiempo completo, prestando cuidados a largo plazo para atender las necesidades de personas mayores con bajo nivel de autonomía, a cambio de un alojamiento gratuito y una compensación económica.

- Una tercera modalidad es la posibilidad de recibir el alojamiento gratuito a cambio de 10 horas semanales de ayuda en tareas cotidianas.

En otros países europeos, las personas alojadoras ofrecen alojamiento a cambio de un precio muy bajo o inexistente. A cambio, las personas alojadas facilitan ayuda, por ejemplo: acompañamiento y pasar la noche en casa; prestar ayuda en casa y en el jardín, cuidar los animales y/o la casa cuando las personas alojadoras están fuera; en Alemania y Austria, una hora de ayuda por cada metro cuadrado de habitación por mes.

\subsection{Criterios de selección}

A) Para las personas alojadoras.

\section{En Reino Unido:}

- Facilitar una habitación independiente.

- Tener una edad comprendida entre los 50 o los 55 años y/o padecer una minusvalía física o sensorial.

- Tener evaluadas e identificadas sus necesidades.

\section{En España, Alemania y Austria:}

- Aceptar la idea del intercambio de ayuda mutua a través del alojamiento compartido.

- La casa debe reunir las condiciones que permitan ofrecer un alojamiento apropiado al estudiante.

- No deben necesitar otro tipo de cuidados. Algunos programas ofrecen sin embargo, este cuidado.

- Deben respetar las horas de estudio de la persona alojada.

- Deben ser personas mayores. Algunos programas extienden la ayuda a familias monoparentales y a personas con problemas de discapacidad. 
B) Para las personas alojadas.

\section{En Reino Unido:}

- Tener una edad mínima de 23 años y ser soltero.

- Ser personas autónomas, trabajadores o estudiantes (para uno de los programas).

- Haber sido entrevistados, evaluados y valoradas sus referencias.

- Comprometerse por un periodo mínimo de 6 meses.

\section{En España, Alemania y Austria:}

- En términos generales, son estudiantes.

- Estar interesados en establecer contacto con personas mayores, o bien tener experiencia como voluntarios.

- Considerar su permanencia en la casa por un periodo mínimo, por ejemplo, igual a un año académico.

- Existen otras limitaciones en lo que respecta a la edad.

3.5. Perfil de las personas alojadoras y de las personas alojadas

\section{Personas alojadoras:}

En todos los países los programas tienen como destinatarios las personas mayores. Alemania y Austria también ofrecen estos programas a familias y familias monoparentales. En España, los programas se dirigieron inicialmente a personas mayores que vivían solas, posteriormente, algunos programas han ido ampliando la figura de la persona alojadora haciendo accesible el programa también a familias, familias con padre o madre solos con personas a su cargo, y a las personas con alguna discapacidad. En Reino Unido también hay programas que van a ampliar su actuación a personas con discapacidad física o de aprendizaje.

En cuanto a la edad de las personas mayores, en Alemania y Austria son personas mayores-jóvenes que pueden valerse por sí mismas, mientras que en España se trata de una población de más edad (media superior a 75 años) con tendencia a un mayor envejecimiento y a necesitar mayor apoyo. En Gran Bretaña, dos de los programas atienden a personas mayores cada vez más jóvenes y tienen proyectado ofrecer el programa a personas menores de 50 años.

La mayoría de las personas mayores son mujeres. En España suele resultar difícil que acepten a estudiantes varones. Sin embargo esto no ocurre así en Alemania donde incluso las mujeres alojadoras valoran las capacidades de los hombres para realizar este tipo de tarea.

Personas alojadas:

En cuanto a las características de las personas alojadas, predomina 
la participación de estudiantes universitarios. En España, las Universidades exigen como requisito ser estudiante universitario. También en Alemania y Austria la mayoría de las veces se alojan estudiantes, por el contrario, en Gran Bretaña suelen ser jóvenes trabajadores aunque también estudiantes. La edad media de estos jóvenes suele oscilar entre 20 y 30 años y, en algunos casos, se exige una edad mínima o máxima para participar.

El número de parejas que se han establecido en cada programa es variable y no se dispone de datos suficientes para realizar la comparación. En general los datos muestran que el número de parejas se ha ido incrementando a lo largo del desarrollo de cada programa. En Alemania es destacable la existencia de parejas que no están incluidas en las estadísticas porque después de haber recibido asesoramiento intensivo de los coordinadores de los programas, son capaces de encontrar pareja por sí mismos. Esto demuestra que el programa promueve realmente la autoayuda.

\subsection{Aspectos que facilitan o dificultan el desarrollo del programa}

El alojamiento compartido ofrece múltiples posibilidades. La idea básica es sencilla y puede ajustarse a muy distintas situaciones, siendo esenciales los conceptos "ayuda mutua" y "solidaridad entre generaciones".

Los programas representan un desafío para quienes los coordinan, que son en su mayoría profesionales de las Ciencias Sociales (trabajadores sociales y psicólogos): Cuanto mejor sea la selección y preparación de las personas que van a participar en el programa, cuanto mejor se consiga satisfacer las necesidades de ambos, persona alojadora y persona alojada, y cuanto mayor sea la competencia profesional de la persona que realiza el seguimiento de las parejas, mayores serán las posibilidades de que las experiencias sean positivas.

El alojamiento compartido estimula e incluso demanda la cooperación de organizaciones e instituciones que representan y trabajan por los intereses de personas mayores y jóvenes.

Las experiencias positivas de convivencia entre las parejas son la mejor forma de promover el programa, difundiendo su éxito entre las personas de su entorno, así como desde los medios de comunicación.

En el momento actual algunos aspectos han frenado una rápida difusión del programa. El principal problema radica en la insuficiente financiación, de modo que no existe dotación de personal cualificado que garantice el desarrollo y calidad de los programas. 
Esta una de las razones por las que es difícil aprovechar y dar respuesta a potenciales usuarios del programa, así como realizar un seguimiento en profundidad de las parejas.

Deben ser eliminados los prejuicios que las personas mayores y sus familiares tienen acerca de los jóvenes, para animarles a formar parte de esta innovadora forma de vivir.

Otras dificultades a nivel estructural son: al inicio del curso existe una mayor demanda de alojamiento por parte de los estudiantes; la demanda de chicas y chicos para alojamiento compartido no es la misma; algunos programas no reúnen la adecuada infraestructura para garantizar un trabajo más eficiente.

\subsection{Sugerencias para personas interesadas en iniciar un progra- ma}

Es necesario que el nuevo programa forme parte de las actividades de una organización o entidad que garantice una infraestructura adecuada para poder ofrecer atención personal y telefónica diaria así como un equipo de profesionales cualificados.

Es importante que se seleccione a profesionales cualificados y que presten la dedicación necesaria para recibir e informar a las personas interesadas, seleccionar cuidadosamente, establecer las parejas más adecuadas y llevar a cabo un seguimiento individualizado y continuo.

Otras personas mayores pueden actuar como voluntarios, colaborando con el equipo de profesionales.

La organización o entidad que desarrolle el programa debe coordinarse con otras instituciones que trabajen con personas mayores y jóvenes.

Hay que invertir tiempo y esfuerzo en la difusión del programa para asegurarse de que su información llega hasta las potenciales personas interesadas. En esta difusión es muy importante el papel de los medios de comunicación.

Los inicios son difíciles. No hay que tener prisa ni esperar grandes resultados a corto plazo. Es mejor asegurarse de las posibilidades de éxito de la convivencia para evitar experiencias negativas en el inicio.

El programa no es la mejor opción para todas las personas. Es un recurso minoritario y es más importante obtener buenos resultados cualitativos que cuantitativos. El objetivo es lograr una buena convivencia intergeneracional y no un registro con un gran número de parejas.

Se puede dirigir la oferta del programa a otras personas que puedan 
precisar en algún momento ayuda y estén dispuestos también a ofrecerla, como personas con discapacidades, padres/madres con personas dependientes a su cargo, y otros.

En definitiva, todas las partes involucradas deben aceptar que se trata de un programa de ayuda mutua y de solidaridad intergeneracional.

\section{CONCLUSIONES}

Todos los países estudiados coinciden en destacar el hecho de que, por una parte, las personas mayores manifiestan que su deseo es continuar viviendo en su propio domicilio en el que suelen tener espacio suficiente para más de una persona. Algunas de estas personas mayores necesitan, o podrían necesitar en el futuro, ayuda para las tareas cotidianas y, en muchos casos, desean vivir en compañía de otra persona para disminuir su soledad. Y, por otra parte, hay evidencia de que muchos jóvenes manifiestan dificultades para satisfacer su necesidad de encontrar un alojamiento a un precio asequible.

Las experiencias de Alojamiento Compartido a Cambio de Ayuda estudiadas realizan actuaciones de mediación entre las personas mayores y los jóvenes para ayudarles a encontrar una respuesta a sus necesidades mediante un intercambio de ayuda mutua, siendo esencial que ambas partes, personas mayores y jóvenes, acepten este intercambio como una ayuda mutua con total libertad.

La creación de estas experiencias y su desarrollo muestran una interesante diversidad. Se advierte una mayor similitud (a pesar de evidentes diferencias) entre los programas de España, Alemania y Austria, lo que parece deberse al hecho de ser iniciativas surgidas desde las Universidades con la intención de promover la solidaridad y la ayuda mutua entre generaciones.

Por ser experiencias innovadoras hay que invertir tiempo en su promoción, con el fin de dar a conocer su existencia a las personas mayores y a los jóvenes. La mejor promoción de estos programas se obtiene mediante la difusión personal que realizan las personas mayores y los jóvenes que han tenido una experiencia de convivencia positiva, así como por la repercusión que ello puede tener en los medios de comunicación.

Las actividades de mediación entre personas mayores y jóvenes (selección, emparejamiento, inicio de la convivencia y seguimiento) deben ser realizadas por profesionales cualificados. Además de estos profesionales, puede ser muy enriquecedor contar con la colaboración de 
personas voluntarias.

Un problema común es el relativo a la financiación de estos Programas. Muchos de ellos tienen problemas para obtener la financiación necesaria que garantice la continuidad de los profesionales cualificados.

Es importante establecer una adecuada coordinación entre la organización que promueve un Programa de Alojamiento Compartido a Cambio de Ayuda y otras organizaciones relacionadas con las personas mayores y con los jóvenes.

En conclusión, podemos destacar dos ideas importantes. En primer lugar, que los Programas de Alojamiento Compartido a Cambio de Ayuda pueden ser un excelente medio para promover la ayuda mutua y la solidaridad intergeneracional. Por lo tanto, son elementos a tener en cuenta en la prevención y la lucha contra la exclusión social de las personas mayores y de los jóvenes. Y, en segundo lugar, los resultados de esta investigación pueden servir para introducir en el Alojamiento Compartido a Cambio de Ayuda a nuevos promotores, a los que se les brinda una magnífica oportunidad de conocer la experiencia de quienes llevan ya tiempo trabajando en este campo.

\section{ANEXO I. PROGRAMAS ESTUDIADOS.}

\section{ESPAÑ}

1. "Convivencia entre Personas Mayores y Jóvenes Estudiantes. Alojamiento Alternativo" Ayuntamiento de Albacete. Albacete.

2. "Alojamiento por Compañía. Programa de Ayuda Mutua entre Personas Mayores y Estudiantes Universitarios". Asociación Universitaria para la Promoción del Voluntariado. Universidad de Alicante. Alicante.

3. "Programa de Alojamiento de Estudiantes Universitarios con Personas Mayores". Universidad de Almería. Almería.

4. "Alojamiento Alternativo: Convivencia Intergeneracional y Ayuda Mutua". Universidad de Deusto. Bilbao.

5. "Programa de Convivencia de Estudiantes con Personas Mayores". Diputación Foral de Guipuzkoa. Departamento de Servicios Sociales. Donostia-San Sebastián.

6. "Programa de Convivencia de Jóvenes Universitarios con Personas Mayores y/o Discapacitadas". Consejería de Asuntos Sociales. Delegación Provincial de Cádiz. Cádiz y Jerez de la Frontera.

7. "Viure y Conviure-Vive y Convive". Caixa de Catalunya-Obra Social. Barcelona, Girona, Lérida, Manresa, Sant Cugat, Cerdanyola del Vallès, 
Tarragona, Terrasa, Vic y Universidades.

8. "Programa de Alojamiento de Universitarios con Personas Mayores y/ o con Discapacitados". Consejería de Asuntos Sociales. Delegación Provincial de Córdoba. Universidad de Córdoba.

9. "Alojamiento Alternativo: Tercera Edad-Estudiantes". Universidad de Granada. Gabinete de Atención Social al Estudiante. Granada.

10. "Servicio de Alojamiento Alternativo". Universidad de Las Palmas de Gran Canaria. Las Palmas de Gran Canaria.

11. "Servicio de Convivencia Ancianos-Universitarios". Escuela Universitaria de Trabajo Social "Ntra. Sra. del Camino". León.

12. "Vivienda Compartida entre Personas Mayores y Estudiantes". Asociación "Solidarios para el Desarrollo"-Universidad Complutense. Madrid.

13. "Programa de Alojamiento de Estudiantes Universitarios con Personas Mayores". Universidad de Málaga. Málaga.

14. "Convivencia entre Generaciones. Programa de Alojamientos Compartidos entre Jóvenes Universitarios y Ancianos de la Región de Murcia". Universidad de Murcia. Murcia.

15. "Convivencia Intergeneracional". Instituto Gerontológico Gallego (IGG). Santiago de Compostela.

16. "Programa de Alojamiento de Universitarios/as con Personas Mayores, Discapacitadas y Padres/Madres con hijos a cargo". Universidad de Sevilla. Sevilla.

17. "Programa de Acercamiento Intergeneracional". Universidad de Valladolid. Valladolid, Palencia, Segovia, Soria.

\section{REINO UNIDO}

1. "Personal Services Society" (PSS). Liverpool (Ruthin, Halton, Kerkintillock).

2. "Homeshare-London". Londres (Hertfordshire, Brighton \& Hove, Bristol, Oxford, Colchester)

3. "Housewise". Northampton.

\section{ALEMANIA}

1. "Wohnen für Hilfe". Universidad de Ciencias Aplicadas de Darmstadt y Cruz Roja. Darmstadt.

2. "Wohnraum für Hilfe". Centro de Personas Mayores (Seniorentreff Neuhausen") y Asociación de Estudiantes de la Universidad de Munich. Munich.

\section{AUSTRIA}

1. "Wohnen für Hilfe". Asociación de Estudiantes Mayores de la Universidad de Graz. Graz 